\title{
Nonergodicity transitions in colloidal suspensions with attractive interactions
}

\author{
J. Bergenholtz \\ Fakultät für Physik, Universität Konstanz, Postfach 5560, D-78457 Konstanz, Germany \\ M. Fuchs \\ Physik-Department, Technische Universität München, 85747 Garching, Germany \\ (Received 9 March 1998; revised manuscript received 22 December 1998)
}

\begin{abstract}
Colloidal gel and glass transitions are investigated using the idealized mode coupling theory (MCT) for model systems characterized by short-range attractive interactions. Results are presented for adhesive hard sphere and hard core attractive Yukawa systems. According to MCT, the former system shows a critical glass transition concentration that increases significantly with introduction of a weak attraction. For the latter attractive Yukawa system, MCT predicts low temperature nonergodic states that extend to the critical and subcritical region. Several features of the MCT nonergodicity transition in this system agree qualitatively with experimental observations on the colloidal gel transition, suggesting that the gel transition is caused by a low temperature extension of the glass transition. The range of the attraction is shown to govern the way the glass transition line traverses the phase diagram relative to the critical point, analogous to findings for the fluid-solid freezing transition. [S1063-651X(99)05905-X]
\end{abstract}

PACS number(s): 64.70.Pf, 82.70.Dd, 64.75. + g, 82.60.Lf

\section{INTRODUCTION}

Attractions among stable colloidal particles lead to a diverse phase behavior. Colloidal attractions, unlike molecular attractions, usually act over a relatively short (compared to the particle size) range. It is by now well established that when the range of the colloidal attraction is decreased the phase diagram undergoes a progression from gas-liquid-solid to fluid-solid coexistence, the latter with a subcooled critical point which is metastable with respect to fluid-solid coexistence [1-5]. Numerous experimental studies show that suspensions often form incompletely equilibrated solids with the appearance of gels where one expects a fluid-solid [6-14] or gas-liquid [15] phase separation from equilibrium theory. The systems studied include mixtures of colloids and nonadsorbing polymer [6-11] and sterically stabilized colloids in marginal solvents [12-17]. In the former case, the attractions stem from a depletion of the polymer coils from the regions between closely spaced particles $[18,19]$, and in the latter case they are caused by surface grafted chain-chain interactions [20]. The gel transition is observed when the range of attraction is short compared to the particle size. In colloidpolymer mixtures this is achieved by choosing a small ratio of polymer to colloid size, whereas the overlap length of the surface grafted chains sets essentially the range of the attraction in the sterically stabilized particle systems.

While the equilibrium phase behavior of these systems is well understood [1-5], the nature of the gel transition remains to be clarified. The gel state appears to be related to a ramified structure with interconnected particle clusters $[10,11]$. Temporal density fluctuations are very slow close to the gel transition, and the suspensions acquire a yield stress and a finite low-frequency elastic shear modulus in the gel $[11,13,14,17,21]$. In the past the transition to the gel state has most often been interpreted as either a static percolation transition $[13,15,22]$, where a sample-spanning cluster of particles forms, or due to the fluid-solid phase transition [14].

Konstanzer Online-Publikations-System (KOPS)

URL: http://www.ub.uni-konstanz.de/kops/volltexte/2007/3671/

URN: http://nbn-resolving.de/urn:nbn:de:bsz:352-opus-36711
Comparison between integral equation predictions for the percolation transition and experimental data, however, shows that the gel transition is confined to the region in the phase diagram between the static percolation threshold and the gasliquid spinodal $[14,15]$. Colloidal gels have also been attributed to states inside a gas-liquid binodal which is metastable with respect to fluid-solid coexistence. Such metastable binodals have indeed been observed for suspensions of globular proteins [23], which also form gels when the ionic strength is sufficiently high [23-25].

In this work we present an alternative interpretation of the dynamical arrest of the gel structure which causes colloidal systems to become disordered solids. We propose that colloidal gels are nonergodic systems that form when a dynamic gel transition is traversed. We further suggest that the gel transition is a low temperature extension of the liquid-glass transition. The gels, however, differ physically from colloidal hard sphere glasses in that they generally display a larger elastic shear modulus, and in that the particles are more strongly localized in the gels; both these effects are due to particle clustering induced by a short-range attraction among particles.

We demonstrate that this is a possible explanation for colloidal gel formation by applying the idealized mode coupling theory (MCT) of the form used to study the liquidglass transition [26-30] to systems in which the attraction is restricted to short ranges. This study is motivated by the observations made by Verduin and Dhont [15], who noted a structural arrest (nonergodicity) in connection with the gel transition similar to that observed for hard sphere colloidal glasses [31-34]. Also Poon et al. [11] made such observations in the so-called transient gelation region of the colloidpolymer phase diagram for short polymers. Krall and Weitz [35] showed that, in the limit of strong particle aggregation, suspensions become nonergodic even at low colloid densities. To date, however, only a speculative connection has been made between the gel and liquid-glass transitions [15]. 
We conduct a study of ergodicity breaking in two model systems: Baxter's adhesive hard sphere (AHS) system and the hard core attractive Yukawa (HCAY) system. Both systems supply analytical solutions for the static structure factor, the former within Percus-Yevick (PY) theory [36], and the latter within the mean spherical approximation (MSA) [37-39]. This study provides more information on the AHS phase diagram and, in addition, serves to complement a recent independent MCT study [40] on the temperature dependence of the AHS glass transition. Further, the HCAY system provides a likely candidate for the gel transition in colloidal systems as an ergodicity breaking dynamic transition of the same type as the liquid-glass transition, suggesting that the experimentally observed gel transition is a low temperature extension of the glass transition.

In what follows, the idealized mode coupling theory of the liquid-glass transition, suitable for colloidal suspensions, is briefly summarized. Results for the temperature dependence of the glass transition are then presented and compared to the AHS phase diagram as predicted by density functional theory. Results are also shown for the HCAY system, which show that the MCT glass transition extends to critical and subcritical regions at low temperatures. The way in which the glass transition line traverses this part of the phase diagram is shown to be a strong function of the range of attraction.

\section{MODE COUPLING THEORY}

The MCT of the liquid-glass transition provides a dynamic description of the transition [26-30,41]. For sufficiently strong interactions the dynamical scattering functions do not decay to zero with time, instead leaving finite residues - the nonergodicity parameters, also known as Edwards-Anderson parameters or glass form factors. Generically, concurrent with this long-time diffusion ceases and the zero-shear viscosity diverges, both due to a diverging relaxation time. This structural relaxation time is in turn related to the particles' inability of escaping their nearest neighbor cages. The glass transition within the framework of the idealized MCT is not a conventional thermodynamic phase transition; the constrained motion of the particles leads to a difference between time and ensemble averages, i.e., an ergodicity breaking transition.

Application of the MCT to the Mori-Zwanzig reduced equations of motion for the density correlators, together with a $t \rightarrow \infty$ limit, leads to the following set of closed equations $[26-30,42]$ :

$$
\begin{gathered}
\frac{f_{q}}{1-f_{q}}=\frac{\rho}{2(2 \pi)^{3} q^{2}} \int d \mathbf{k} V(\mathbf{q}, \mathbf{k})^{2} S_{q} S_{k} S_{|\mathbf{q}-\mathbf{k}|} f_{k} f_{|\mathbf{q}-\mathbf{k}|}, \\
V(\mathbf{q}, \mathbf{k})=\hat{\mathbf{q}} \cdot(\mathbf{q}-\mathbf{k}) c_{|\mathbf{q}-\mathbf{k}|}+\hat{\mathbf{q}} \cdot \mathbf{k} c_{k}+q \rho c^{(3)}(\mathbf{q}, \mathbf{q}-\mathbf{k}), \\
\frac{f_{q}^{s}}{1-f_{q}^{s}}=\frac{\rho}{(2 \pi)^{3} q^{2}} \int d \mathbf{k} V^{s}(\mathbf{q}, \mathbf{k})^{2} S_{k} f_{k} f_{|\mathbf{q}-\mathbf{k}|}^{s},
\end{gathered}
$$

where $\rho$ is the number density, $S_{q}$ is the static structure factor, $c_{q}=\left(S_{q}-1\right) / \rho S_{q}$ is the Fourier-transformed OrnsteinZernike direct correlation function, and $c^{(3)}$ is the triplet direct correlation function. In this study we use primarily the so-called convolution approximation $\left(c^{(3)}=0\right)[27,29,43]$ for the triplet direct correlation function. Note that the coupling vertices $V(\mathbf{q}, \mathbf{k})$ and $V^{s}(\mathbf{q}, \mathbf{k})$ in Eqs. (1) and (2) are free of singularities and vary smoothly with a set of external control parameters, e.g., the particle density. The coherent $\left(f_{q}\right)$ and incoherent $\left(f_{q}^{s}\right)$ nonergodicity parameters are defined as the long-time limits of the intermediate scattering function $F_{q}(t)$ and the self-intermediate scattering function $F_{q}^{s}(t)$, according to

$$
\begin{gathered}
f_{q}=\lim _{t \rightarrow \infty}\left(F_{q}(t) / S_{q}\right), \\
f_{q}^{s}=\lim _{t \rightarrow \infty}\left(F_{q}^{s}(t)\right) .
\end{gathered}
$$

The nonergodicity parameters determine the properties of the glass. The zero-frequency elastic shear modulus of the colloidal glass (in units of $k_{B} T / \sigma^{3}$, with $k_{B} T$ the temperature and $\sigma$ the particle diameter) is given by $[27,44]$

$$
G=\frac{\sigma^{3}}{60 \pi^{2}} \int_{0}^{\infty} d k k^{4}\left(\frac{d \ln S_{k}}{d k} f_{k}\right)^{2} .
$$

The incoherent nonergodicity parameter $f_{q}^{s}$ is found to be well approximated by a Gaussian, the half-width of which is proportional to the mean-square displacement in the glass state [26,27]. The localization length $r_{s}$ is defined as the root-mean-square displacement in the glass, and is determined from $f_{q}^{s}=1-q^{2} r_{s}^{2}$ for $q \rightarrow 0$ [27].

Equations (1) and (2) are solved self-consistently for the nonergodicity parameters as functions of specified external control parameters: the reduced temperature $\tau$ and volume fraction $\phi=\pi \rho \sigma^{3} / 6$ for the AHS system, and the reduced temperature $K^{-1}$, screening parameter $b$, and volume fraction for the HCAY system (cf. below). The solution proceeds by iteration, first on $f_{q}$, and subsequently on $f_{q}^{s}$. Transition lines delineating ergodic and nonergodic states were found by bracketing, and the monotonicity property of the iteration [45] was employed. The integrations were performed numerically using Simpson's rule on a uniformly discretized wave vector grid: $q_{i}=i \Delta q, i=0, \ldots, N$. The parameters $\Delta q$ and $N$ used varied somewhat, but most results were obtained using $0.15<\Delta q \sigma<0.30$ and $600<N<1000$. The iterative solution scheme was complemented occasionally by an algorithm to speed up convergence by using stored previous iterates [46]. We have also directly integrated the equations of motion [47] (adjusted to obey Smoluchowski dynamics). This yields the entire time evolution of the density correlators $F_{q}(t)$ and $F_{q}^{s}(t)$, the long-time limits of which were found to be identical to the solutions of Eqs. (1) and (2).

The result $f_{q}=f_{q}^{s}=0$ is always a solution to Eqs. (1) and (2), implying that correlations among density fluctuations vanish for long times. At low densities this is the only solution, hence the system is in a fluid, possibly metastable fluid state. Above a critical volume fraction $\phi_{c}$ also nonzero solutions appear, which correspond to nonergodic glass states.

$$
V^{s}(\mathbf{q}, \mathbf{k})=\hat{\mathbf{q}} \cdot \mathbf{k} c_{k},
$$




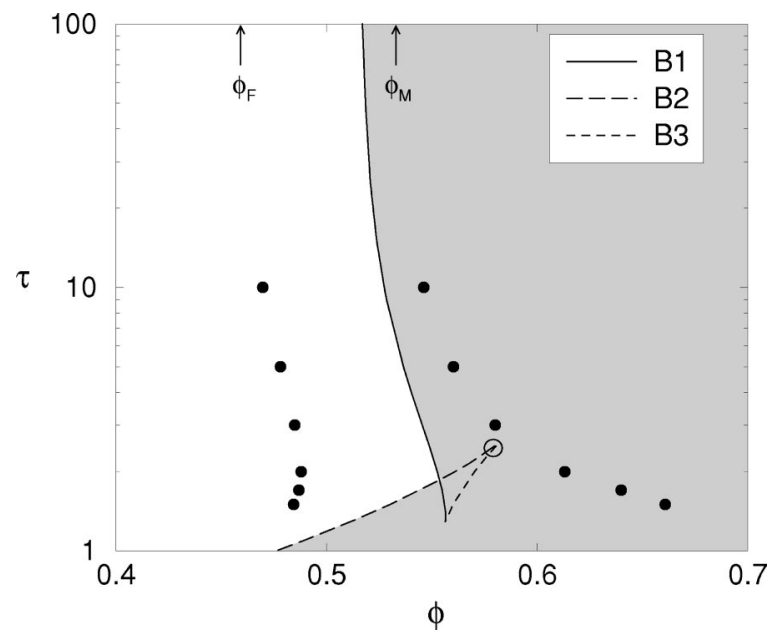

FIG. 1. AHS phase diagram in terms of the reduced temperature $\tau$ and the particle volume fraction $\phi$. The labeled lines are the bifurcation lines of Eq. (1) discussed in the text. The shaded region encloses nonergodic density fluctuations. Liquid-glass transitions occur when crossing the $B 1$ and $B 2$ lines into the shaded nonergodic region. Glass-glass transitions occur along the piece of the $B 2$ line inside the nonergodic region. The physically relevant end point singularity is indicated by a circle. The data for the fluid-solid freezing and melting lines $(\bullet)$ are taken from MWDA calculations [55]. The arrows show the (PY) hard sphere freezing $\left(\phi_{F}\right)$ and melting $\left(\phi_{M}\right)$ volume fractions [55].

The physical solutions to Eqs. (1) and (2), corresponding to the long-time limits defined by Eqs. (3) and (4), were identified by choosing the largest solutions for $f_{q}$ and $f_{q}^{s}[27,45]$.

\section{ADHESIVE HARD SPHERE SYSTEM}

The AHS pair potential consists of an infinitely deep and narrow well located at particle contact, given explicitly by [36]

$$
u(r) / k_{B} T=\lim _{d \rightarrow \sigma^{+}}\left\{\begin{array}{l}
\infty, \quad 0<r<\sigma \\
\ln \left[\frac{12 \tau(d-\sigma)}{d}\right], \quad \sigma<r<d \\
0, \quad d<r,
\end{array}\right.
$$

where $\tau$ is a reduced temperature and $r$ is the interparticle separation distance. The $\tau \rightarrow \infty$ limit of the PY-AHS system corresponds to the PY hard sphere system. Using this as the starting point, the earlier MCT result for the hard sphere glass transition volume fraction $\phi_{c}=0.516$ [26] was reproduced. With more accurate hard sphere static inputs one obtains $\phi_{c}=0.525[27,48,49]$. Experiments locate the colloidal hard sphere glass transition at $\phi_{c} \approx 0.58$ [31-34], showing that the idealized MCT prediction for the hard sphere $\phi_{c}$ is too low; this is presumably caused by the strong restriction of the modes of structural relaxation imposed in the MCT.

The locus of critical glass transition points is shown in Fig. 1 as a function of the particle volume fraction $\phi$ and the reduced temperature $\tau$, with the shaded region denoting nonergodic glass states. Upon decreasing $\tau$ the glass transition point moves along the line $B 1$ in Fig. 1 to higher density, contrary to the findings for particles interacting via a molecu- lar interaction potential of Lennard-Jones form [49]. We see that starting with a hard sphere glass and introducing a shortrange attraction leads to an ergodicity restoring transition, provided the density of the isochore is not too high. The glass transition for $\tau=2$ occurs at a volume fraction of 0.5527 , significantly higher than the MCT hard sphere result. The strength of attraction needed to shift the glass transition to higher density is weak; a second virial coefficient mapping reveals that a reduced temperature $\tau=2$ corresponds roughly to a $0.05 \sigma$ wide square well with a depth of $\sim 0.6 k_{B} T$. An examination of the static structure factors along the critical glass transition boundary $B 1$ in Fig. 1 shows that they are not markedly different from those of hard sphere suspensions; however, subtle changes in the structure lead to significant changes in the critical glass transition density.

At high temperatures we observe that the MCT predictions for the localization length $r_{s}$ follow roughly a Lindemann criterion, given by the hard sphere result $r_{s} \approx 0.074 \sigma$, in agreement with the results of the Lennard-Jones study [49]. Below $\tau \sim 5$, however, the Lindemann criterion is violated, the particles now being more strongly localized in the glass state. We note also that inclusion of improved triplet correlations in the manner of Ref. [48], who used an approximation due to Denton and Ashcroft for $c^{(3)}$ [50], leads to a qualitatively similar phase diagram with boundaries shifted slightly to lower densities and temperatures relative to those shown in Fig. 1.

The AHS phase behavior has been the subject of several studies, most of them using density functional theory (DFT) [51-55]. Selecting the most recent one by Marr and Gast [55] for comparison, who used the modified weighteddensity approximation (MWDA) [56], we find that the $B 1$ glass transition is confined to the metastable region between the fluid-solid coexistence lines (see Fig. 1). One striking feature is that the $B 1$ glass transition line from MCT and the MWDA freezing transition line track each other, the quantity $\Delta \phi=\phi_{c}-\phi_{f}$, with $\phi_{f}$ the volume fraction at freezing, being nearly temperature independent.

This result has interesting consequences for the diffusion constants at the freezing densities [30,57]. When sufficiently close to the glass transition, the long-time self-diffusion coefficient assumes its asymptotic behavior governed by the distance to the glass transition singularity. The normalized long-time self-diffusion coefficient has been found to exhibit universality along the fluid-solid freezing transition [58]. The comparison made here shows that this condition may be related to a universality of the proximity of the freezing transition to the glass transition. At least, it suggests a deeper connection between MCT for the liquid-glass transition and DFT, a topic that has been explored to some extent [59].

In following the glass transition line from high to low temperatures (the line denoted by $B 1$ in Fig. 1), we find a line crossing similar to that studied within schematic ( $q$ independent) models $[27,47,60]$. This region in the phase diagram was studied in detail recently by Fabbian et al. [40]. At the crossing between the $B 1$ and $B 2$ glass transition lines, it is $B 2$ that determines the behavior of the physical solution, as the nonergodicity parameters associated with $B 2$ are found to be always greater than those associated with $B 1$. Thus, along the $B 2$ line bordering the fluid phase, $f_{q}$ for each $q$ jumps discontinuously between 0 and finite values, and 
between smaller and larger finite values when the $B 2$ line is traversed in the glass. The appearance of the $B 2$ line is a result of an end point (cusp, A3) singularity $[27,40,60,61]$ in the AHS phase diagram, where three solutions of Eq. (1) for $f_{q}$ coalesce. This singularity appears as the termination point of the $B 2$ transition line. It is connected to another bifurcation point with triply degenerate $f_{q}$ solutions located at lower temperature by the $B 3$ transition line shown in Fig. 1. Neither the low temperature end point, the piece of $B 1$ between it and $B 2$, nor the $B 3$ transition line play a role in determining the glass dynamics, but show the connectivity among the bifurcation solutions of Eq. (1).

The $B 2$ line in Fig. 1 exhibits unusual properties. Varying the numerical parameters $N$ and $\Delta q$, such that the maximum wave vector $q_{\max }=N \Delta q$ changes, shifts the location of the $B 2$ glass transition line and the high temperature end point in the phase diagram. Such a variation is not observed in connection with the $B 1$ glass transition line. In addition, we were unable to identify a set of $N$ and $\Delta q$ such that the $f_{q}$ associated with the $B 2$ glass transition decays to zero within the prescribed wave vector range. The results shown in Fig. 1 were obtained using $N=700$ and $\Delta q \sigma=0.2$.

It is possible that the anomalous behavior of the $B 2$ glass solutions results from the atypical behavior of the AHS $S_{q}$ in the large- $q$ limit caused by the singular nature of the AHS pair potential. The AHS $S_{q}$ decays slowly for large $q$ as $S_{q}$ $\approx 1+2 \phi \lambda_{B} \sin (q \sigma) / q \sigma$, where $\lambda_{B}$ is the solution of Baxter's quadratic equation [36]. We do not consider the behavior of the $B 2$ glass solutions here further; instead, we show in Sec. IV that the HCAY system exhibits glass transition lines that extend to low temperatures and densities. Several properties of these low temperature glasses are in qualitative agreement with experiments on colloidal gels.

\section{HARD CORE ATTRACTIVE YUKAWA SYSTEM}

In this section we examine the effect on the glass transition of introducing a finite range of attraction via the HCAY system. The HCAY pair potential is given by

$$
u(r) / k_{B} T=\left\{\begin{array}{l}
\infty, \quad 0<r<\sigma \\
-\frac{K}{r / \sigma} e^{-b(r / \sigma-1)}, \quad \sigma<r,
\end{array}\right.
$$

where the dimensionless parameter $K$ regulates the depth of the attractive well and the reduced screening parameter $b$ sets the range of the attraction. Using the MSA static structure factor [37-39] as input, the MCT was solved for three different screening parameters: $b=7.5,20$, and 30 . The progression of the glass transition can be traced from the (PY) hard sphere limit, corresponding to $K=0$, to lower temperatures in terms of the reduced temperature $K^{-1}$.

In Fig. 2 we show the gas-liquid spinodal curves, studied in detail by Cummings and co-workers [39,62]. The critical temperature is sensitive to the range of the attraction, decreasing with increasing $b$. The spinodal curves are shown as indicators of where gas-liquid phase coexistence will occur, should there be a stable liquid phase. Included in the diagrams in Fig. 2 are the corresponding loci of glass transition points. At high temperatures and small values of $b(b$ $=7.5$ ) they are relatively insensitive to the strength of the

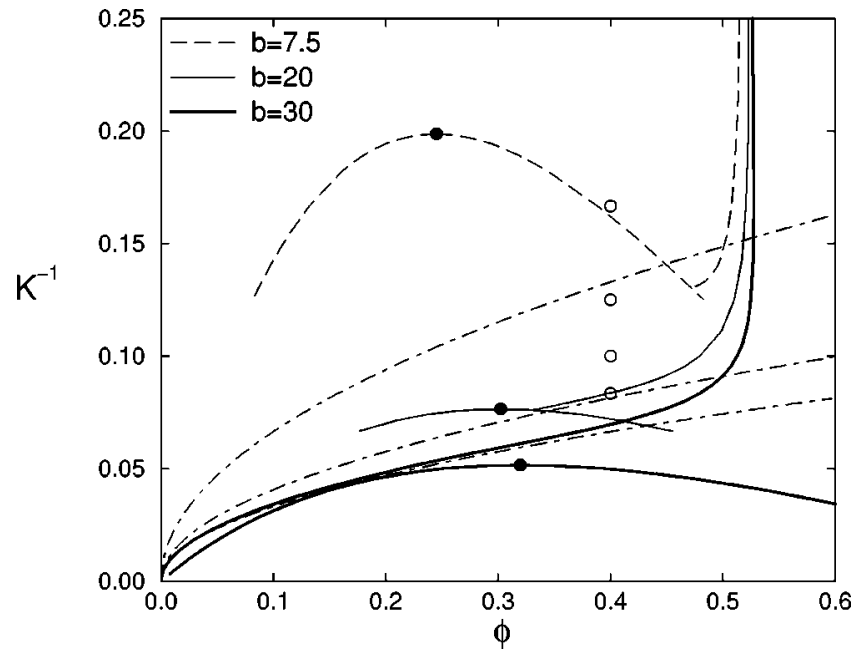

FIG. 2. HCAY phase diagrams in terms of the reduced temperature $K^{-1}$ and particle volume fraction $\phi$ for varying values of the screening parameter $b$, as labeled. MSA gas-liquid spinodals are shown with critical points denoted by $(\mathbf{O})$, together with the corresponding MCT glass transition lines as labeled. The chain curves correspond to the asymptotic prediction in Eq. (8) with $\Gamma_{c}(b \rightarrow \infty)$ $=3.02$. The open circles $(\bigcirc)$ denote the locations in the $b=20$ diagram of the suspensions for which the results in Fig. 6 were calculated.

attraction, showing only a minor initial movement toward higher densities. Increasing the value of the screening parameter $b(b=20$ and 30$)$, which decreases the range of the attraction, leads to a small initial increase of the glass transition density upon lowering the temperature, although this trend is not as pronounced as in the AHS system; subsequently, at lower temperatures the glass transition is induced at increasingly lower densities. For $b=7.5$ and 20 the nonergodicity transition lines reach subcritical temperatures, and approach the liquid side of the spinodal. For $b=30$ the nonergodicity transition line lies entirely within the fluid phase above the two phase region, and extends to subcritical temperatures at low densities.

The MCT used here does not account for large concentration gradients and additional critical slowing of relaxations. As there is no small expansion parameter in MCT, it is difficult to ascertain when, upon approaching a critical point, this form of MCT should be replaced by a more complete theory, including a more sophisticated handling of the critical dynamics (see Ref. [63] and references therein). At high temperatures, wave vectors around the primary peak of the structure factor contribute the most to the mode coupling integrals in Eqs. (1) and (2). With decreasing temperature, on the one hand, the small wave vector structure in $S_{q}$ leads to a stronger coupling on large length scales; on the other hand, the attractive interactions become of increasing importance on all length scales. The former effect, which can be expected to appear for all ranges of attractions, leads to nonergodicity transitions which trace the spinodal lines. These transitions will be discussed in the Appendix, as here the present MCT is least reliable because it does not include all relevant mode couplings and will not correctly describe the dynamics near the critical points [63]. The latter effect, important for systems with short-range attractions, can be studied by an asymptotic analysis of the MCT equations and will 
be seen to dominate the low density glass transitions for large values of $b$.

At low densities and in the limit of strong attractive interactions, the Ornstein-Zernike direct correlation function becomes independent of density. Specifying this to the MSA of the HCAY system, this limit corresponds to $\phi \rightarrow 0$ and $K$ $\rightarrow \infty$. Considering the asymptotic limits

$$
\phi \rightarrow 0 \quad \text { and } K \rightarrow \infty \text {, }
$$

so that

$$
\Gamma=\frac{K^{2} \phi}{b}=\text { const }
$$

the MCT equations simplify because $S_{q} \rightarrow 1$ follows. The nonergodicity transitions then occur at $\Gamma=\Gamma_{c}(b)$, leading to the asymptotic prediction $K_{c} \propto 1 / \sqrt{\phi_{c}}$.

For short-range attractions, in the limit of $b \rightarrow \infty$, a further simplification arises because the coupling constant $\Gamma$ approaches a unique value at the transition $\Gamma_{c} \rightarrow 3.02$ for $b$ $\rightarrow \infty$, and the nonergodicity parameters now depend only on the rescaled wave vector $\tilde{q}=q \sigma / b: f_{q}^{c} \rightarrow \tilde{f}^{c}(\tilde{q})$. The asymptotic transition lines are shown in Fig. 2 as the chain curves. We find excellent agreement with the MCT transition line for $b=30$ at low density, demonstrating that the low density nonergodicity transitions are not driven by the divergence of the small wave vector limit of $S_{q}$. Moreover, the asymptotic model is seen to capture the behavior of the full MCT transition lines-where present-qualitatively and even semiquantitatively at higher densities.

We further point out that Eq. (2) for the single particle dynamics and, thus, the incoherent form factors $f_{q}^{s}$ are not dominated by small wave vector variations in the static structure factor. Instead, $f_{q}^{s}$ and the localization length $r_{s}$ are dominated by the small distance or large wave vector behavior of the liquid structure. In the asymptotic limit of Eq. (8) this also holds for the collective particle dynamics, and $\tilde{f}^{c}(\tilde{q})=\widetilde{f}^{s}(\tilde{q})$ is obtained, where both functions show rather large non-Gaussian corrections.

For the system with $b=20$, Fig. 2 shows that the glass transition nearly meets the critical point (see also Fig. 7 in the Appendix). This aspect is in qualitative agreement with the behavior of the sterically stabilized suspensions studied by Verduin and Dhont [15]. They observed a gel transition that traversed the phase diagram from high density and temperature to the critical point. The transition, which they refer to as a static percolation transition, was associated with a nondecaying intermediate scattering function and nonfluctuating dynamic light scattering speckle patterns. Thus it has the expected properties of the ergodic-nonergodic dynamic transition predicted by the idealized MCT. Moreover, they were able to follow the transition into the unstable region inside the spinodal curve, showing that complete phase separation does not occur because of interference from the gel transition. We cannot extend the calculation of the MCT gel transition line into the unstable region because an appropriate $S_{q}$ is not available, and the theory assumes closeness to equilibrium [29].

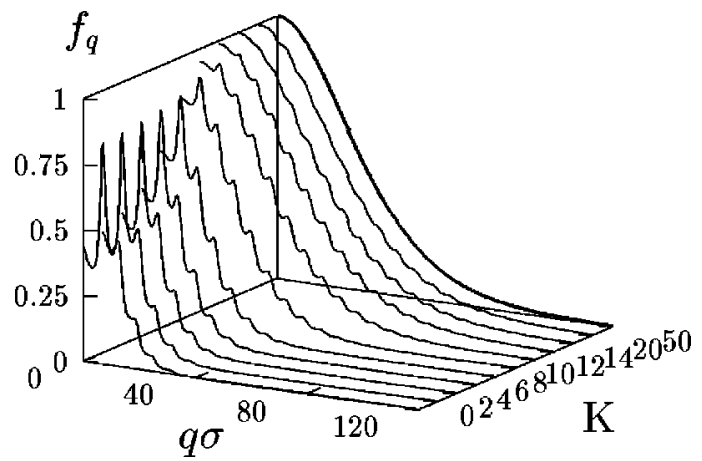

FIG. 3. HCAY critical coherent nonergodicity parameters $f_{q}$ for $b=30$ as functions of normalized wave vector $q \sigma$ and Yukawa prefactor $K$. Note that the volume fraction varies according to the $b=30$ critical glass transition line in Fig. 2, and that the $K$ values jump from $K=14-20$ and 50 . The rear curve shown in bold is the density and temperature independent asymptotic prediction resulting from Eq. (8) and $b \rightarrow \infty$.

Restricting the range of the attraction sufficiently, as for $b=30$, Fig. 2 shows that the glass transition line passes above the critical point and reaches subcritical temperatures on the gas side of the metastable spinodal. For such systems we may speculate that the glass transition renders the entire spinodal curve and the liquid phase dynamically inaccessible. This feature appears to agree with some measurements on sterically stabilized suspensions [13,14,17], in which only a liquid-gel transition was observed.

Recent measurements by Poon et al. [11] suggested that the colloid-polymer mixtures with a small polymer/colloid size ratio $(\xi \approx 0.08)$ may belong to the class of HCAY phase diagrams with $b<20$, where the nonergodicity transition line meets the spinodal on the liquid side. This interpretation includes a possible explanation for the growth of the smallangle scattering peak for samples with low colloid concentrations [11], and that the denser colloid domains arrest in the transient gelation region.

To further clarify the physical mechanism of the gel transition and the properties of the gel states, various aspects of the solutions of the MCT will be discussed for three cases: $b=7.5,20$, and 30. In Fig. 3 we show the evolution of the coherent nonergodicity parameter $f_{q}$ along the critical glass transition boundary corresponding to $b=30$ in Fig. 2. As seen, the width of $f_{q}$ increases with decreasing temperature (increasing $K$ ). This behavior of $f_{q}$ with decreasing temperature is a result of a corresponding increase in the range of $S_{q}$, which results from particles being strongly correlated near contact, i.e., due to particle clustering. For longer range attractions, like the $b=7.5$ system, the width of $f_{q}$ and $f_{q}^{s}$ remain essentially unchanged along the glass transition boundary, which reflects the lower degree of particle clustering in this system. Note that $f_{q}$ becomes a density and temperature independent function, $f_{q} \rightarrow \widetilde{f}(q \sigma / b)$, in the limit of strong attractions. This prediction is shown in Fig. 3 as the bold line, and agrees almost quantitatively with the full MCT $f_{q}$ solutions for large values of $K$.

The localization length $r_{s}$ in the glass decreases along the glass transition boundary when the attraction is sufficiently short range. This decrease in the localization length is shown in Fig. 4, and is caused by the increased contribution from 


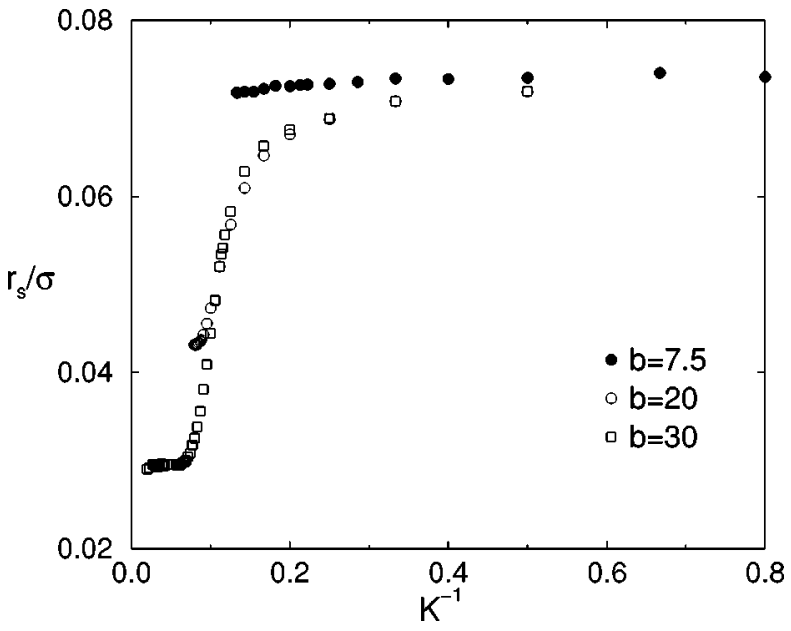

FIG. 4. Localization length (root-mean-square displacement) $r_{s}$ in the glass along the critical glass transition lines in Fig. 2 as a function of the reduced temperature $K^{-1}$ for various values of the Yukawa screening parameter $b$, as labeled.

large wave vectors in the MCT integrals in Eqs. (1) and (2). For longer range attractions, like the $b=7.5$ case, the localization length stays close to the value dictated by the Lindemann criterion and found at the glass transition in the hard sphere system $[26,49]$. Thus for short-range attractions the particles are more strongly localized in the glass than for systems with somewhat longer range attractions. At low temperatures the localization length saturates at a limiting value which is inversely proportional to $b$ because of Eq. (8), which leads to the prediction $r_{s} \rightarrow 0.91 \sigma / b$ for $b \rightarrow \infty$.

In addition to an increased width at low temperatures, the small wave vector behavior of $f_{q}$ changes dramatically, such that at low temperatures the intermediate scattering function for small $q$ practically does not decay with time at all (see Fig. 3). This indicates that large scale assemblies of particles behave essentially as static objects, where the single particles are tightly bound to the particle clusters (see Fig. 4). The asymptotic limit, Eq. (8) and $b \rightarrow \infty$, which results in $\widetilde{f}(\tilde{q})$ $-1 \propto \tilde{q}^{2}$, stresses that this is caused by the short-range attraction. Such a rise in $f_{q}$ for small $q$ is observed also in the $b$ $=7.5$ system, where it is caused by a different mechanism, namely the increase in the isothermal compressibility on approaching the gas-liquid spinodal. There this leads to coherent nonergodicity parameters which are essentially hardsphere-like except for a large $q \rightarrow 0$ value.

In Fig. 5 we show the zero-frequency elastic shear modulus as a function of the reduced temperature along the glass transition lines in Fig. 2. When the range of attraction is comparatively large $(b=7.5)$ the shear modulus remains constant at the hard sphere value, even for suspensions near the spinodal. This illustrates that the shear modulus, like the localization length, is determined by the large wave vector behavior of the liquid structure $S_{q}$, and is unaffected by long-wavelength density fluctuations in our calculations. For shorter range attractions $(b=20$ and 30$)$, the shear modulus is dominated by particle clustering; it increases strongly with decreasing temperature because of the stronger binding among particles, eventually showing a maximum for suspensions close to the bend in the $K_{c}$ versus $\phi_{c}$ curves, where $\phi_{c}$ begins to decrease strongly with decreasing temperature. At

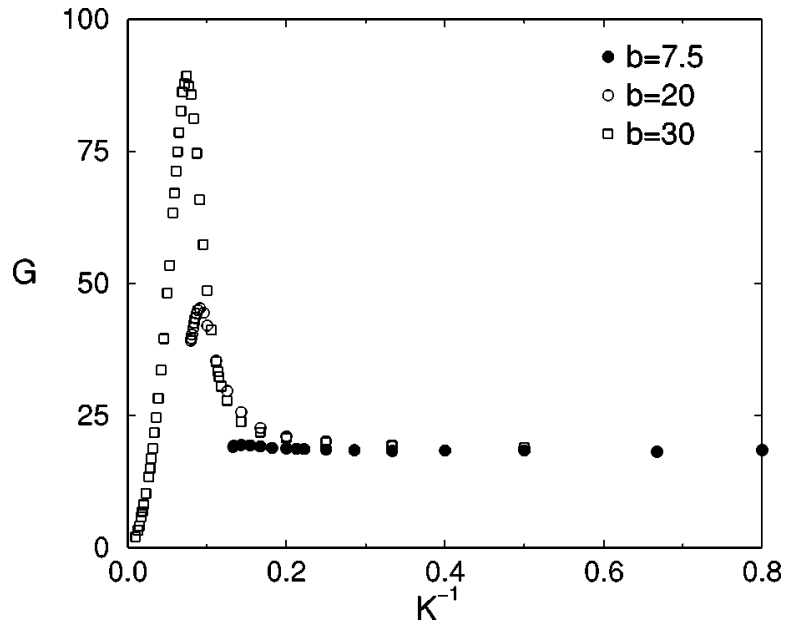

FIG. 5. Elastic shear modulus (in units of $k T / \sigma^{3}$ ) in the glass along the critical glass transition lines in Fig. 2 as a function of the reduced temperature $K^{-1}$ for various values of the Yukawa screening parameter $b$, as labeled.

lower density the shear modulus becomes linear in the density at the transition, according to $G \propto K_{c}^{2} \phi_{c}^{2} / b$, as predicted by the asymptotic solution in Eq. (8), and as observed in the dilute limit of the $b=30$ system. These results show that low temperature nonergodic structures, proposed to be colloidal gels here, are distinct from colloidal glasses in that they generally display a larger static shear modulus and strongly localized particles bound in clusters.

To connect this study of the low temperature behavior of the glass transition more clearly to the experimental studies of the gel transition, we have calculated the intermediate scattering function upon approaching the glass transition at fixed volume fraction. This mimics the Verduin and Dhont study [15], in which they performed low- $q$ dynamic light scattering experiments on a series of suspensions at fixed volume fraction close to the gel transition. As noted already, the HCAY system with $b=20$ exhibits a glass transition line that nearly meets the critical point. This qualitative aspect is shared with the experimental phase diagram determined by Verduin and Dhont. We have selected four suspensions with $b=20$ and $\phi=0.4$ at different reduced temperatures (shown as open circles in Fig. 2), such that the suspension with the lowest temperature $(K=12)$ is located in the glass.

The resulting normalized intermediate scattering functions corresponding to these suspensions are displayed in Fig. 6 for a fixed wave vector $q \sigma=0.2$, the same wave vector as that used in the measurements by Verduin and Dhont. As the normalized intermediate scattering function is the quantity that one measures in dynamic light scattering experiments, we can compare Fig. 6 with the results of Verduin and Dhont (see their Fig. 11). This comparison shows excellent qualitative agreement between their dynamic light scattering data and our calculated $F_{q}(t) / S_{q}$. Away from the transition the decay of $F_{q}(t) / S_{q}$ is approximately exponential for this wave vector. The decay becomes slower when the temperature is decreased until $K=12$, when $F_{q}(t) / S_{q}$ no longer decays to zero. Instead, a long-time plateau with a value near unity is obtained, which corresponds to the nonergodicity parameter $f_{q}$ at $q \sigma=0.2$. Note that additional incoherently scattered light due to particle size polydispersity 


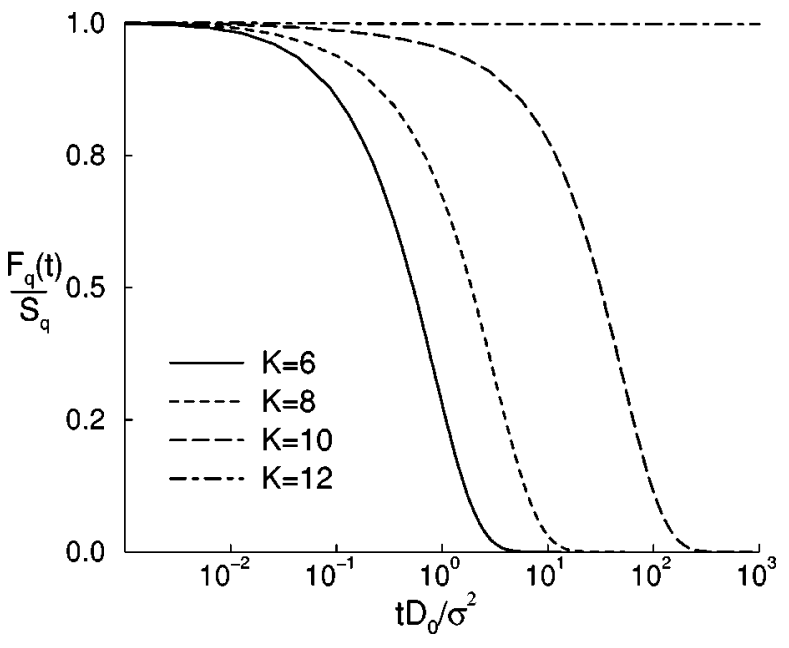

FIG. 6. Normalized intermediate scattering function at a fixed wave vector $q \sigma=0.2$ as a function of Yukawa prefactor and dimensionless time, as labeled. The corresponding locations in the $b$ $=20$ HCAY diagram are shown in Fig. 2 as open circles.

[15] may contribute appreciably and cause $f_{q}$ to attain such a large value. Nevertheless, the dynamical arrest of $F_{q}(t) / S_{q}$ agrees with our proposed ergodic-nonergodic transition for the gel transition and is captured by the idealized MCT.

As has been shown in the past, the range of the colloidal attraction dictates where the fluid-solid freezing transition passes through the phase diagram and whether there is a stable liquid phase [1-5]. In the same manner, Fig. 2 shows that the range of the attraction determines how the glass transition traverses the phase diagram relative to the critical point. We have not compared the diagrams in Fig. 2 with results for the fluid-solid and gas-liquid transitions (see, e.g., Refs. $[4,64,65])$. The MCT relies on the static structure factor input, which was provided using the MSA. For shortrange attractions the MSA produces relatively poor structural and thermodynamic predictions. Thus a fair comparison should be made with a theory, e.g., the MWDA [56], which can use the same input as that supplied to the MCT. Alternatively, the MCT can be solved using a more accurate static structure factor, such as that from HMSA theory [66]. This would enable a determination of the location of the glass transition relative to that of the fluid-solid freezing transition, testing the conjecture made here that the glass transition line tracks the freezing transition at higher density in the phase diagram.

\section{DISCUSSION AND CONCLUSIONS}

The idealized MCT has been shown to provide a possible explanation for important aspects of the colloidal gel transition. In this scenario the arrest of the dynamics during the gel transition is caused by a low temperature liquid-glass transition. The underlying phenomenon is a breaking of ergodicity, caused by long-time structural arrest. It is accompanied by the cessation of hydrodynamic diffusion and the appearance of relatively large finite elastic moduli as the particles are tightly localized in ramified clusters.

The AHS system was found to have end-point singularities in the phase diagram. The spectacular dynamics close to the MCT end-point singularity has been the focus of a recent study [40]. However, the glass transitions that occur at low temperatures in the AHS system are accompanied by numerical difficulties, resulting from the singular nature of the AHS pair interaction potential. Nevertheless, the AHS system provides insight into the temperature dependence of the glass transition in systems with weak short-range attractions. Subtle changes in the structure caused by the attractions lead to an initial increase in the glass transition density with decreasing temperature. The particles forming the glassy cage tend to stick together, thereby creating openings in the collective cage around a central particle which have to be filled by increasing the critical colloid density. We suggest that the recrystallization of glass samples at high densities upon addition of short polymers, reported in Ref. [8], is explained by this shift of the glass transition density to higher values. Moreover, the MCT glass transition line was observed to lie parallel to the DFT fluid-solid freezing transition at high temperatures in the AHS phase diagram.

Introduction of a finite range of attraction, and replacement of the PY theory with the MSA via the HCAY system, yields glass transition lines that extend to low temperatures in the phase diagram. For HCAY systems with moderaterange attractions the glass transition line crosses the liquid binodal. When the range of the attraction is further restricted, the glass transition line passes above the critical point, likely rendering part of the (metastable) equilibrium phase diagram irrelevant. Preliminary solutions of the dynamical MCT equations for the $b=30$ HCAY system indicate that nearby $A_{l}$ singularities with $l>2$ appreciably distort the time dependent structural correlators in the intermediate time windows, even though no $A_{3}$ singularity $[27,29,40,61]$ could be found in the phase diagram.

The nonergodicity transitions of the HCAY system are influenced by two mechanisms which are absent or not dominant in the hard sphere and Lennard-Jones [49] systems. In the latter two, the temperature dependence of the critical density $\rho_{c}$ is either trivially absent or arises from the soft repulsive part of the pair interaction potential. Along the MCT transition line in the Lennard-Jones liquid, the temperature dependent packing fraction $\phi(\rho, T)$, resulting from the effective excluded volume diameter $\sigma=\sigma^{\text {eff }}(T)$ [67], is roughly constant $\phi(\rho, T) \approx 0.52$ and approximately equal to its hard sphere value [49]. As the soft repulsion of the Lennard-Jones system leads to $\sigma^{\text {eff }} \propto T^{-1 / 12}$, the critical density smoothly decreases with temperature [68]. Note that this observation indicates that the nonergodicity transitions resulting from the solution of Eq. (1) for the Lennard-Jones system are dominated by the excluded volume effect, i.e., the primary peak of the structure factor $S_{q}$, as is also the case for the hard sphere system. Because the idealized MCT has been developed from approximations aimed at describing the connected physical mechanism, called cage- or back-flow effect, the quality of the mode coupling approximation is expected to be unaffected by temperature changes as long as these excluded volume effects dominate in Eqs. (1) and (2).

The nonergodicity transition lines of the HCAY system, on the other hand, are additionally affected by the low wave vector fluctuations in the fluid structure factor, $S_{q}$ for $q \rightarrow 0$, and by the increase in $S_{q}$ at large wave vectors arising from the short-range nature of the attraction. The first aspect, which also occurs for longer range attractions, leads to non- 
ergodicity transitions tracking the spinodal curve (see the Appendix).

The short-range nature of the attraction causes the stronger localization of the particles, i.e., the shorter localization length $r_{s}$, upon decreasing the temperature. Also, the strong increase in the elastic shear moduli along the nonergodicity transition line occurs only for sufficiently short-range attractions, as shown in Fig. 5. Again, the ability of the MCT equations (1) and (2) to describe such local interparticle correlations is not known. Note, however, that the HCAY results are independent of the numerical parameters chosen. Clearly, theories aimed at long-wavelength phenomena at the gel transition cannot incorporate these variations of the elastic modulus as described by the MCT because it arises from local potential energy considerations.

The asymptotic model, defined by Eq. (8) (and $b \rightarrow \infty$ ), which highlights the effects of strong short-range attractions, captures all aspects of the low density MCT nonergodicity transitions qualitatively and even semiquantitatively. Furthermore, it clearly demonstrates that the gel transitions are not driven by long-range structural correlations. It can be expected that such an asymptotic model can be found for other theories of liquid structure with strong short-range attractive potentials, but the detailed predictions presented here rest upon the use of the MSA for the HCAY system.

Based on this suggested interpretation of the MCT nonergodicity transitions, several features of the computed HCAY density-temperature diagrams agree qualitatively with experimental observations made on colloid-polymer mixtures and sterically stabilized suspensions [6-14,16,17]. First, the gel transitions appear to lie at lower temperatures than, but otherwise track, the freezing line when present. Second, for short-range attractions the gel transition can shift to comparable or higher temperatures than those required for gasliquid phase separation. Third, the gel line does not show such a strong density dependence as the static percolation transition.

We emphasize that this suggested interpretation of the colloidal gel transitions is based on an extension of the idealized MCT of the glass transition beyond the range its approximations were aimed at. Our speculation, however, can be decisively tested by dynamic light scattering experiments. Nonergodicity transitions within the MCT exhibit universal dynamical properties $[27,29,61,69]$, which, for example, led to the identification of the colloidal hard sphere glass transition by van Megen and co-workers [31-34]. As more complicated bifurcation scenarios, $A_{l}$ with $l>2[27,29,40,61]$, can be expected, the dynamics at the gel transitions should be very nonexponential and anomalous. Moreover, the shortrange attractions lead to couplings among more wave vector modes, as can be seen from the asymptotic model defined by Eq. (8), resulting in a MCT exponent parameter (see Refs. $[27,69]$ for a definition and details on its calculation) $\lambda$ $=0.89$ for $b \rightarrow \infty$, considerably larger than values found for systems not characterized by short-range attractions (see, e.g., Refs. [40,48,68]).

The proposed connection between the MCT nonergodicity transitions in the HCAY system and the nonequilibrium transitions in colloidal suspensions is further supported by the following observations. For moderate-range attractions, like the $b=7.5$ diagram in Fig. 2, the coexistence region can be tentatively divided into three regions. For somewhat lower temperatures than the critical temperature, gas-liquid phase separation occurs, provided a thermodynamically stable liquid phase exists. For temperatures (just) below the triple point temperature, gas-crystal phase separation takes place. Decreasing $K^{-1}$ still more, gas-glass coexistence may be expected if - as argued from computer simulations [70]-the way to crystallization proceeds via the initial formation of a liquid droplet, whose density lies above the nonergodicity transition line. As the glass states for this system are rather close to the ideal hard sphere glass state, we expect signatures of this well studied transition to be observed $[28,29,31-34]$. We speculate that the vanishing of the homogeneously nucleated crystallites in the colloid-polymer systems upon addition of sufficient large molecular weight polymer, observed in Ref. [9], signals the presence of a nonergodicity transition as found in the colloidal hard sphere system $[34,71,72]$. This suggestion can be tested by studying the dynamic density fluctuations close to the transition, as has been demonstrated in the hard sphere system [28,29,3134].

For suspensions with short-range attractions, like the $b$ $=20$ or 30 curves in Fig. 2, it seems possible that the longrange density fluctuations, likely induced by the hidden critical point, become arrested when the denser domains of the system cross the MCT nonergodicity transition line. Nonergodic gel states characterized by large small wave vector form factors $f_{q}$, rather short localization lengths, and finite, rather large elastic moduli can be expected. We suggest that these nonergodicity transitions cause the gel transitions observed in the colloid-polymer mixtures and sterically stabilized suspensions, and anticipate that they may also play a role in other colloidal systems, such as emulsions [73], emulsion-polymer mixtures [74], and suspensions of globular proteins [23-25], in which short-range attractions also dominate. We caution again that a proper extension of the MCT used here to include a full description of the critical dynamics close to critical points has yet to be formulated.

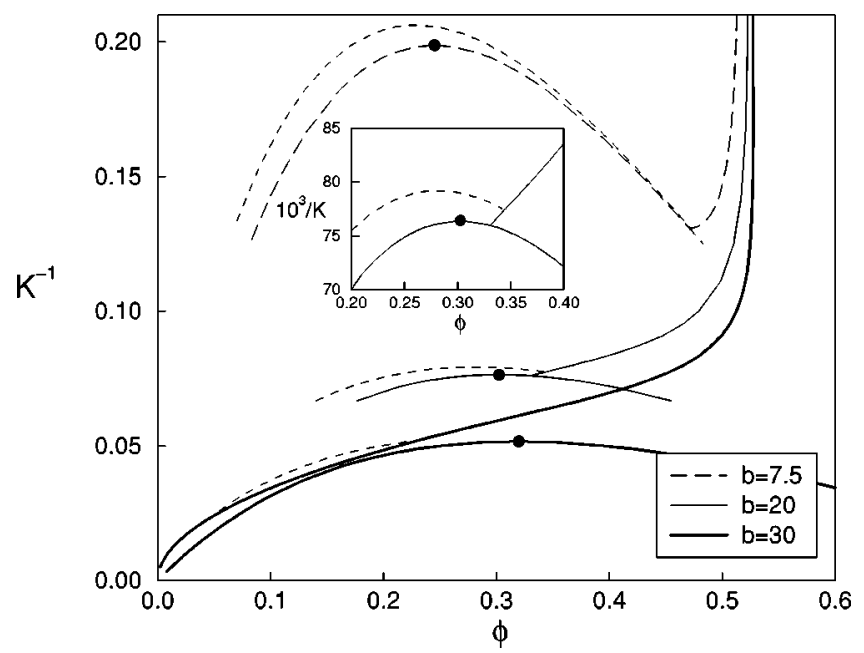

FIG. 7. HCAY phase diagrams showing, in addition to the MSA gas-liquid spinodal curves and the MCT glass transition lines as in Fig. 2, the MCT transition lines (short-dashed lines) which are characterized by a small- $q$ structural arrest in $f_{q}$ and $f_{q}^{s}=0$. The inset shows an enlargement of the line crossing in the vicinity of the $b$ $=20$ critical point. 
Experimental tests of the dynamics close to the gel transitions would be required to test our suggestion.

\section{ACKNOWLEDGMENTS}

J.B. acknowledges financial support from the National Science Foundation (Grant No. INT-9600329) and the kind hospitality of Professor R. Klein (Universität Konstanz). The work was further supported by the Deutsche Forschungsgemeinschaft (DFG) (Grant No. Fu309/2-1). Useful discussions with G. Nägele and N. J. Wagner are acknowledged. We also thank Fabbian et al. [40] for making their work available to us prior to publication.

\section{APPENDIX}

In this appendix the nonergodicity transitions caused by the increase in the $q \rightarrow 0$ limit of the structure factor close to the spinodal lines are discussed for the HCAY system. Figure 7 shows the spinodal lines and the gel transitions for the interaction parameters considered in Secs. IV and V. Also shown are nonergodicity transition lines occurring only close to the spinodal lines. As seen, there is at least one crossing of the two types of nonergodicity transitions for each attraction range $b$, where in all cases the gel transitions discussed in the main text provide the larger, physical nonergodicity parameters. The additional transition lines presented here in the Appendix have two peculiarities which cause us to doubt the validity of the present MCT for their description. First, they are directly caused by the small wave vector structure in $S_{q}$; thus a proper MCT description should also include the very likely present critical dynamics [63]. Second, at these transition lines only the collective density fluctuations for exceedingly small wave vectors or on large length scales are arrested. The single particle dynamics remain fluidlike, i.e., $f_{q}^{s}=0$ from Eq. (2). Again, this suggests that long-range collective fluctuations are of crucial importance at these transitions, and that the simple MCT approach used here is likely insufficient.
[1] A. P. Gast, C. K. Hall, and W. B. Russel, J. Colloid Interface Sci. 96, 251 (1983).

[2] A. P. Gast, W. B. Russel, and C. K. Hall, J. Colloid Interface Sci. 109, 161 (1986).

[3] C. F. Tejero, A. Daanoun, H. N. W. Lekkerkerker, and M. Baus, Phys. Rev. Lett. 73, 752 (1994).

[4] M. H. J. Hagen and D. Frenkel, J. Chem. Phys. 101, 4093 (1994).

[5] H. N. W. Lekkerkerker, J. K. G. Dhont, H. Verduin, C. Smits, and J. S. van Duijneveldt, Physica A 213, 18 (1995).

[6] P. D. Patel and W. B. Russel, J. Colloid Interface Sci. 131, 192 (1989).

[7] S. Emmett and B. Vincent, Phase Transit. 21, 197 (1990).

[8] W. C. K. Poon, J. S. Selfe, M. B. Robertson, S. M. Ilett, A. D. Pirie, and P. N. Pusey, J. Phys. II 3, 1075 (1993).

[9] S. M. Ilett, A. Orrock, W. C. K. Poon, and P. N. Pusey, Phys. Rev. E 51, 1344 (1995).

[10] N. A. M. Verhaegh, D. Asnaghi, H. N. W. Lekkerkerker, M. Giglio, and L. Cipelletti, Physica A 242, 104 (1997).

[11] W. C. K. Poon, A. D. Pirie, M. D. Haw, and P. N. Pusey, Physica A 235, 110 (1997).

[12] J. W. Jansen, C. G. de Kruif, and A. Vrij, J. Colloid Interface Sci. 114, 481 (1986).

[13] M. Chen and W. B. Russel, J. Colloid Interface Sci. 141, 564 (1991).

[14] M. C. Grant and W. B. Russel, Phys. Rev. E 47, 2606 (1993).

[15] H. Verduin and J. K. G. Dhont, J. Colloid Interface Sci. 172, 425 (1995).

[16] C. J. Rueb and C. F. Zukoski, J. Rheol. 41, 197 (1997).

[17] C. J. Rueb and C. F. Zukoski, J. Rheol. 42, 1451 (1998).

[18] S. Asakura and F. Oosawa, J. Chem. Phys. 22, 1255 (1954).

[19] A. Vrij, Pure Appl. Chem. 48, 471 (1976).

[20] P. W. Rouw and C. G. de Kruif, J. Chem. Phys. 88, 7799 (1988)

[21] H. Verduin, B. J. de Gans, and J. K. G. Dhont, Langmuir 12, 2947 (1996).

[22] A. T. J. M. Woutersen, J. Mellema, C. Blom, and C. G. de
Kruif, J. Chem. Phys. 101, 542 (1994).

[23] M. Muschol and F. Rosenberger, J. Chem. Phys. 107, 1953 (1997).

[24] A. George and W. W. Wilson, Acta Crystallogr., Sect. D: Biol. Crystallogr. 50, 361 (1994).

[25] D. Rosenbaum, P. C. Zamora, and C. F. Zukoski, Phys. Rev. Lett. 76, 150 (1995).

[26] U. Bengtzelius, W. Götze, and A. Sjölander, J. Phys. C 17, 5915 (1984).

[27] W. Götze, in Liquids, Freezing and Glass Transition, edited by J.-P. Hansen, D. Levesque, and J. Zinn-Justin (North-Holland, Amsterdam, 1991), p. 287.

[28] W. Götze and L. Sjögren, Phys. Rev. A 43, 5442 (1991).

[29] W. Götze and L. Sjögren, Rep. Prog. Phys. 55, 241 (1992).

[30] M. Fuchs, Transp. Theory Stat. Phys. 24, 855 (1995).

[31] P. N. Pusey and W. van Megen, Phys. Rev. Lett. 59, 2083 (1987).

[32] W. van Megen and P. N. Pusey, Phys. Rev. A 43, 5429 (1991).

[33] W. van Megen and S. M. Underwood, Phys. Rev. E 49, 4206 (1994).

[34] W. van Megen, Transp. Theory Stat. Phys. 24, 1017 (1995).

[35] A. H. Krall and D. A. Weitz, Phys. Rev. Lett. 80, 778 (1998).

[36] R. J. Baxter, J. Chem. Phys. 49, 2770 (1968).

[37] E. Waisman, Mol. Phys. 25, 45 (1973).

[38] J. S. Hoye and L. Blum, J. Stat. Phys. 16, 399 (1977).

[39] P. T. Cummings and E. R. Smith, Chem. Phys. 42, 241 (1979).

[40] L. Fabbian, W. Götze, F. Sciortino, P. Tartaglia, and F. Thiery, Phys. Rev. E 59, R1347 (1999).

[41] E. Leutheusser, Phys. Rev. A 29, 2765 (1984).

[42] G. Szamel and H. Löwen, Phys. Rev. A 44, 8215 (1991).

[43] H. W. Jackson and E. Feenberg, Rev. Mod. Phys. 34, 686 (1962).

[44] G. Nägele and J. Bergenholtz, J. Chem. Phys. 108, 9893 (1998).

[45] W. Götze and L. Sjögren, J. Math. Anal. Appl. 195, 230 (1995).

[46] K.-C. Ng, J. Chem. Phys. 61, 2680 (1974). 
[47] M. Fuchs, W. Götze, I. Hofacker, and A. Latz, J. Phys.: Condens. Matter 3, 5047 (1991).

[48] J. L. Barrat, W. Götze, and A. Latz, J. Phys.: Condens. Matter 1, 7163 (1989).

[49] U. Bengtzelius, Phys. Rev. A 33, 3433 (1986).

[50] A. R. Denton and N. W. Ashcroft, Phys. Rev. A 39, 426 (1989).

[51] C. Cerjan and B. Bagchi, Phys. Rev. A 31, 1647 (1985).

[52] S. J. Smithline and A. D. J. Haymet, J. Chem. Phys. 83, 4103 (1985).

[53] X. C. Zeng and D. W. Oxtoby, J. Chem. Phys. 93, 2692 (1990).

[54] C. F. Tejero and M. Baus, Phys. Rev. E 48, 3793 (1993).

[55] D. W. Marr and A. P. Gast, J. Chem. Phys. 99, 2024 (1993).

[56] A. R. Denton and N. W. Ashcroft, Phys. Rev. A 39, 4701 (1989).

[57] M. Fuchs, Phys. Rev. Lett. 74, 1490 (1995).

[58] H. Löwen, T. Palberg, and R. Simon, Phys. Rev. Lett. 70, 1557 (1993).

[59] T. R. Kirkpatrick and P. G. Wolynes, Phys. Rev. A 35, 3072 (1987).

[60] W. Götze and R. Haussmann, Z. Phys. B 72, 403 (1988).
[61] W. Götze and L. Sjögren, J. Phys.: Condens. Matter 1, 4203 (1989).

[62] P. T. Cummings and G. Stell, J. Chem. Phys. 78, 1917 (1983).

[63] K. Kawasaki, in Phase Transitions and Critical Phenomena, edited by C. Domb and M. S. Green (Academic, New York, 1976).

[64] M. Renkin and J. Hafner, J. Chem. Phys. 94, 541 (1991).

[65] M. Hasegawa, J. Chem. Phys. 108, 208 (1998).

[66] G. Zerah and J.-P. Hansen, J. Chem. Phys. 84, 2336 (1986).

[67] J. D. Weeks, D. Chandler, and H. C. Andersen, J. Chem. Phys. 54, 5237 (1971).

[68] M. Nauroth and W. Kob, Phys. Rev. E 55, 657 (1997).

[69] W. Götze, Z. Phys. B 60, 195 (1985).

[70] P. R. ten Wolde and D. Frenkel, Science 277, 1975 (1997).

[71] W. van Megen and S. M. Underwood, Nature (London) 362, 616 (1993).

[72] J. L. Harland and W. van Megen, Phys. Rev. E 55, 3054 (1997).

[73] J. Bibette, T. G. Mason, H. Gang, and D. A. Weitz, Phys. Rev. Lett. 69, 981 (1992).

[74] A. Meller, T. Gisler, D. A. Weitz, and J. Stavans, Langmuir 15, 1918 (1999). 